\title{
The Trends of Psychological Status of People Entering from High-Risk Areas of COVID-19 Coronavirus During the Quarantine in Dedicated Hotels: A Longitudinal Survey Study from Guangzhou, China
}

Yanhong Deng, (iD) ${ }^{1} *$ Lishuo Shi, (iD) $2 *$ Jianxia Li, (iD) ${ }^{1} *$ Zhipeng Jiang, ${ }^{3} *$ Chaojun Xie, ${ }^{4}$ Shuangling Luo, ${ }^{3}$ Li Ling, ${ }^{5}$ Hualiang Lin, ${ }^{6}$ Zongqiu Chen, ${ }^{4}$ Yunlong Zhao, ${ }^{7}$ Liang Kang, (D) ${ }^{3}$ Jun Yuan, ${ }^{4}$ Weiping Wen ${ }^{8}$

'Department of Medical Oncology, The Sixth Affiliated Hospital, Sun Yat-sen University, Guangdong Provincial Key Laboratory of Colorectal and Pelvic Floor Disease, Guangzhou, 51 0655, People's Republic of China; ${ }^{2}$ Clinical Research Center, The Sixth Affiliated Hospital, Sun Yat-sen University, Guangdong Provincial Key Laboratory of Colorectal and Pelvic Floor Disease, Guangzhou, 510655 , People's Republic of China; ${ }^{3}$ Department of Colorectal Surgery, The Sixth Affiliated Hospital, Sun Yat-sen University, Guangdong Provincial Key Laboratory of Colorectal and Pelvic Floor Disease, Guangzhou, 510655 , People's Republic of China; ${ }^{4}$ Guangzhou Center for Disease Control and Prevention, Guangzhou, Guangdong Province, 510440, People's Republic of China; ${ }^{5}$ Department of Medical Statistics, School of Public Health, Sun Yat-sen University, Guangzhou, 51 10080, People's Republic of China; ${ }^{6}$ Department of Epidemiology, School of Public Health, Sun Yat-sen University, Guangzhou, 510080 , People's Republic of China; ${ }^{7}$ Medical Department, the Sixth Affiliated Hospital, Sun Yat-sen University, Guangdong Provincial Key Laboratory of Colorectal and Pelvic Floor Disease, Guangzhou, 510655, People's Republic of China; ${ }^{8}$ Department of Otolaryngology, the Sixth Affiliated Hospital, Sun Yat-sen University, Guangdong Provincial Key Laboratory of Colorectal and Pelvic Floor Disease Guangzhou, 510655, People's Republic of China

*These authors contributed equally to this work

Correspondence: Weiping Wen

Department of Otolaryngology, The Sixth

Affiliated Hospital of Sun Yat-sen University,

Guangdong Provincial Key Laboratory of

Colorectal and Pelvic Floor Disease, No. 26

Yuan Cun Er Heng Road, Guangzhou, 510655,

People's Republic of China

Tel +86-20-87333733

Email wenwp@mail.sysu.edu.cn

Jun Yuan

Guangzhou Center for Disease Control and Prevention, No. I, Qide Road of Baiyun

District, Guangzhou, Guangdong Province,

51 0440, People's Republic of China

Tel +86-20-36052333-18I6

Email yuanjuncom@I63.com
Background: The quarantine in dedicated hotels has become an inevitable safety measure due to the frequent cross-border travel since the outbreak of COVID-19. The aim of the present study was to explore the trends in the psychological status of individuals entering from high-risk areas of COVID-19 coronavirus while quarantining in dedicated hotels.

Methods: A total of 591 individuals who isolated in dedicated hotels were recruited between March and June 2020. Participants self-reported mental symptoms [Self-rating Anxiety Scale (SAS) and Self-rating Depression Scale (SDS)] every three days during the quarantine. A mixed-effects linear regression model was used to assess the trends.

Results: Participants reporting anxiety and depression symptoms at least one time during quarantine accounted for $4.5 \%$ and $18.4 \%$, respectively. Their psychological status was alleviated during some first 9 days, and then it slightly deteriorated, which was suggested by SAS and SDS scores that were negatively correlated with the days of quarantine (T) (adjusted coefficient $[\beta]-0.81,95 \% \mathrm{CI}-1.00$ to -0.62 ; and $\beta-0.75,95 \% \mathrm{CI}-0.97$ to -0.53 , respectively), and were positively correlated with the square of $\mathrm{T}$ ( $\beta 0.04,95 \% \mathrm{CI} 0.02$ to 0.06 ; and $\beta 0.04,95 \%$ CI 0.02 to 0.06 , respectively). The unemployed and 18 30-year-old participants were prone to greater levels of psychological distress. No significant difference in the trend of mental health was found among different subgroups.

Conclusion: The mental health of the people entering Guangzhou from high-risk areas of COVID-19 coronavirus resulted positive during the early period of quarantine in dedicated hotels, after which it deteriorated. The psychological status of individuals should be closely monitored at the beginning and after more than 9 days of quarantine, especially for individuals who are unemployed and 18 30-year-old ones.

Keywords: quarantine, dedicated hotels, psychological distress, COVID-19

\section{Introduction}

In December 2019, there was an outbreak of the coronavirus disease 2019 (COVID19) in China that rapidly swept across the world. By April 2021, there were more than 136 million confirmed cases globally. ${ }^{1}$ Due to the long incubation period of COVID-19 and the fact that covert cases could represent some $60 \%$ of all infections, ${ }^{2}$ quarantining assists in separating and restricting the movement of people who were potentially exposed to the disease and is thought to effectively control the spread of the disease. ${ }^{3}$ 
People entering from high-risk areas of COVID-19 coronavirus are usually required to be self-isolated at home or quarantined in dedicated facilities for 14 days. However, the infection can be easily transmitted to other family members due to inadequate prevention and control measures of home quarantine,${ }^{4,5}$ thus, quarantine in dedicated facilities was advocated in many countries or regions when the infectious disease broke out. ${ }^{3}$ Following the overwhelming global spread of the COVID-19 epidemic in the second half of 2020, many countries or regions besides mainland China, such as Hong Kong, ${ }^{6}$ Australia, ${ }^{7}$ Singapore, ${ }^{8}$ and even the England, ${ }^{9}$ have started to use dedicated quarantine hotels for isolation of people entering from high-risk areas or countries. Following these policies, a large number of people were quarantined in dedicated facilities due to cross-border travel. Eg, in Hong Kong, ${ }^{10}$ about 100 thousand person-times left for mainland China in December 2020 alone, and about 10 thousand people entered from high-risk areas other than mainland China.

Restriction of activities and isolation might affect people's psychological health. COVID-19 has been reported to cause a series of psychological health threats in the general public. ${ }^{11-13}$ In particular, people who had quarantining experiences during the epidemic outbreak had more psychological distress than those who did not. ${ }^{14,15}$ In addition, most of the previous studies on the impact of quarantining on individual's mental health were cross-sectionally designed and were comparing the mental health status of people with and without isolation experience, ${ }^{14-17}$ while few studies have reported on dynamic psychological changes in quarantined individuals in dedicated hotels throughout the period of a couple of weeks. Such an approach may help us identify more effective mental health improvement strategies. Even though the governments have generally realized that psychological counseling is needed for quarantined individuals, it remains unclear when might be the best time point for psychological counseling and which subgroups are the most vulnerable to psychological stress. As the environment people face (people quarantining at home vs people quarantining in the hotel) is quite different, ${ }^{3}$ it is necessary to separately explore the mental health of people quarantining in dedicated hotels. Therefore, exploring the dynamic psychological changes and their influencing factors is of great importance. It might help us formulate more effective and targeted psychological intervention measures, especially if we consider that the number of individuals who are quarantined in dedicated hotels will continue to grow due to the frequent cross-border travel.

Hence, the aim of this study was to longitudinally evaluate the status of depression and anxiety during the quarantine period in dedicated hotels by using a questionnaire survey in a time-dependent manner.

\section{Methods}

\section{Study Design and Participants}

This longitudinal study was conducted in Guangzhou, Guangdong province of China, between March 31 and June 10, 2020. It has been reported that $90 \%$ of people enter China through Guangdong Province, and nearly 30,000 people are quarantined in dedicated hotels in Guangdong Province every day. ${ }^{18}$ Guangzhou is the capital of Guangdong Province, and as such, it has faced great pressure in epidemic import during the epidemic period of 2019-nCoV.

We used cluster sampling to identify two dedicated quarantine hotels, after which the volunteers were recruited to participate in this study. According to the length of the incubation period of 2019-nCoV, quarantine usually lasts 14 days. All participants voluntarily participated in the project, and they were asked to fill out the questionnaires via the online survey tool Wenjuanxing (https://www.wjx.cn/app/survey.aspx) once every three days. The process diagram is shown in Figure 1. A total of 591 individuals participated in our study, and 1691 valid

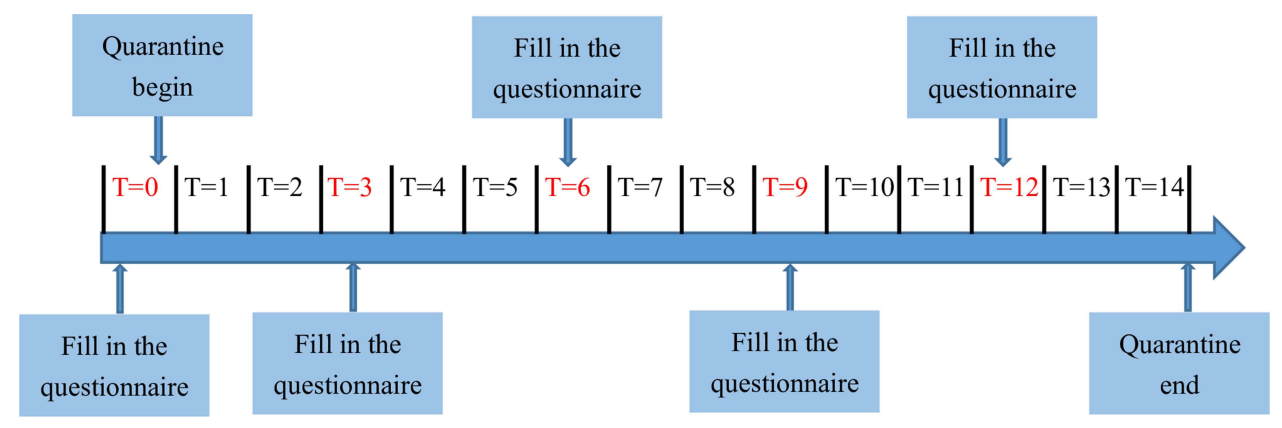

Figure I The process diagram of participants after enrollment. All participants were advised to fill out the questionnaire every three days until the end of the quarantine. 
questionnaires were collected. Before filling the questionnaire, written consent was obtained from all participants during the first section of the online survey. If a participant was under 18 years old, informed consent was obtained from respective guardians. The diagram of enrollment of participants during this project is shown in Figure A1 in Supplement. The local ethics committee provided ethics approval.

\section{Procedures}

The psychological status of the participants was measured by the self-rating anxiety scale (SAS) ${ }^{19}$ and self-rating depression scale (SDS) ${ }^{20}$ Both scales consisted of 20items and were based on a 4-point Likert scale ranging from 1 ("not at all") to 4 ("almost always yes"). After multiplying the total score of the questionnaire by 1.25 , the standard scale score ranged between 25 and 100, with the higher scores referring to a higher likelihood of having anxiety and depression. The two questionnaires have been widely used in Chinese populations ${ }^{21,22}$ and were reported to have good validity and reliability (The Cronbach's alphas of SAS and SDS were 0.85 and 0.91, respectively). ${ }^{23,24}$ In the present study, the Cronbach's alpha of SAS and SDS was 0.808 and 0.885 , respectively. The two scales also provided a grading method, where the standard scores of SAS and SDS $<50$ points indicated no anxiety and depression, respectively; the standard score of 50 to 60 points indicated mild symptoms; 61 to 70 points indicated moderate symptoms; and $>70$ points indicated severe symptoms.

We focused on the changing trends in the psychological status of the individuals during the quarantine period; thus, the days of quarantine $(\mathrm{T})$ when the questionnaire was submitted was used as the independent variable. Each participant was required to fill out the questionnaire on day $1(\mathrm{~T}=0)$ before beginning the quarantine and to continue to fill it out at subsequent time points $(\mathrm{T}=3,6,9 \ldots)$ throughout the quarantine.

Based on literature review, seven putative confounders that covered demographic, socioeconomic, and environmental were chosen. ${ }^{3,25-28}$ Demographic variables including gender (male and female), age (under $18,18-30,31-40,41-50$, and $\geq 51$ years), and place of departure (domestic and international transportation) were extracted. Socioeconomic variables, ie, job (student, employed, or unemployed) and education (secondary school and below, college degree, university-Bachelor, university-Master and above) were extracted.
Environmental variables included the environment of the room (whether light is sufficient or not) and the exact date the questionnaire responses were submitted. This was important because, with the change of epidemic situation, different date period was related to different epidemic situation and information about the virus, which may also have an impact on the participants' mental health. As these variables might be related to the mental health of the participants during quarantine, they were included as covariates.

Two following multiple-choice questions were included in the questionnaire to invite participants to voluntarily answer at the end of the quarantine: "the reasons why you feel disturbed during the quarantine period"; "what information can relieve your uneasiness during the quarantine period".

\section{Statistical Analysis}

The confounders were described, and analysis of variance was used to compare the scale scores among different groups. Longitudinal SAS and SDS scores were used as dependent variables in multilevel mixed-effects linear regression models with maximum likelihood estimation and were implemented in SPSS23.0. Compared with the traditional repeated measurement analysis of variance, the mixed effect model does not require the same time interval for each response of the sample and is less affected by the missing data at some time points. ${ }^{29}$ In this study, data missing at some time points of the scales were not filled in. Mean scores were used for the outcome measure rather than fitting a model for a binary outcome indicator. This is because such a model approach could further reduce the sample available for analysis as it excludes those with concordant responses over follow-up when exploring the fixed effect of independent variables, affecting the statistical power and generalizability of the findings. This analysis nests correlates the data, thereby accounting for violations in the assumption of independence. For the present data, repeated assessments over time were nested in individuals (the random effect). Fixed effects (ie, predictors in the regression) included linear, quadratic effects of days of quarantine ( $\mathrm{T}$ and $\mathrm{T}^{2}$ ) and confounders. Interactions between the days of quarantine and predefined subgroups were fitted to investigate heterogeneity in the effect of days of quarantine on mental health. Effect estimates were also reported by subgroup, and the associated $p$ values were used to test the null hypothesis, ie, that there is no evidence for a difference in the effect of days of 
quarantine on mental health between different subgroups of participants.

A sensitivity analysis was done to assess the potential impact of some participants who did not fill the questionnaire at recommended time points. For this analysis, the mixed-effects analysis was re-run after the data were revised as follows: the questionnaire filled out on days 2 and 4 of quarantine ( $\mathrm{T}=2$ and 4 ) was adjusted to the day 3 ( $\mathrm{T}=3$ ), on days 5 and 7 ( $\mathrm{T}=5$ and 7) was adjusted to day 6 ( $\mathrm{T}=6)$, on 8 and 10 days ( $\mathrm{T}=8$ and 10) was adjusted to day $9(\mathrm{~T}=9)$, and on 11 and above days $(\mathrm{T} \geq 11)$ was adjusted to day $12(\mathrm{~T}=12)$.

\section{Results}

A total of 591 individuals participated in the study, and 1691 questionnaires were collected. Among the participants, 51.3\% were males, and $48.7 \%$ were females, with age ranging from 18-30 years (65.1\%). Students accounted for $57.0 \%$, and the proportion of undergraduates was the highest (42.1\%). Moreover, $65.8 \%$ of the participants entered Guangzhou via international transportation, as shown in Table 1. The same person filled out the questionnaire maximally 6 times and at least once. The majority of the people filled out the questionnaire 3 times, accounting for $27.24 \%$, as shown in Table A1 in Supplement. Individuals reported anxiety and depression symptoms at least once during the whole quarantine period, accounting for $4.5 \%$ and $18.4 \%$, respectively. With the progression of quarantine, the incidence of anxiety and depression showed no significant change $(\mathrm{P}=0.890$ and 0.562 , respectively) (Tables A2 and A3 in Supplement).

At the beginning of quarantine $(\mathrm{T}=0)$, female participants, aged 31-40, the unemployed, and people entering Guangzhou by domestic transportation had higher scores of SAS or SDS than others, as shown in Table 1. Among 1691 questionnaires, significant differences were also found in the scores of SAS or SDS among different characteristics of the participants (Table A4 in Supplement). Also, the scores of the scales were significantly different between the questionnaires submitted at different days of quarantine and different dates (Table A5 in Supplement).

Figure 2 showed that with increased days of quarantine, the SAS and SDS scores of the participants had a downward

Table I Demographic Characteristics and Standard Scale Scores of Participants in Baseline (T=0)

\begin{tabular}{|c|c|c|c|c|c|}
\hline Variables & $n(\%)$ & SAS(SD) & $P$ value & SDS(SD) & P-value \\
\hline Gender & & & 0.37 & & 0.0012 \\
\hline Male & $303(51.3)$ & $34.46(7.68)$ & & $36.67(10.75)$ & \\
\hline Female & $288(48.7)$ & $35.01(7.27)$ & & $38.59(11.11)$ & \\
\hline Age & & & 0.013 & & 0.23 \\
\hline Under 18 & $76(12.9)$ & $32.11(7.84)$ & & $35.38(11.73)$ & \\
\hline $18 \sim 30$ & $385(65.1)$ & $35.10(7.13)$ & & $38.01(10.63)$ & \\
\hline $31 \sim 40$ & $66(11.2)$ & $36.00(7.8 I)$ & & $36.57(10.79)$ & \\
\hline $4 \mid \sim 50$ & $39(6.6)$ & $33.94(8.05)$ & & $38.01(12.48)$ & \\
\hline $5 \mathrm{I} \sim$ & $25(4.3)$ & $34.85(8.57)$ & & $40.10(11.12)$ & \\
\hline Education & & & 0.12 & & 0.23 \\
\hline Secondary school and below & $165(27.9)$ & $34.43(8.4 I)$ & & $37.80(11.86)$ & \\
\hline College degree & $56(9.5)$ & $37.03(7.52)$ & & $39.62(11.66)$ & \\
\hline University-Bachelor & $249(42.1)$ & $34.52(6.91)$ & & $36.98(10.24)$ & \\
\hline University-Master and above & $86(14.6)$ & $34.8 I(6.89)$ & & $36.06(8.93)$ & \\
\hline Missing & $35(5.9)$ & & & & \\
\hline Job & & & 0.0050 & & 0.0018 \\
\hline Student & $337(57.0)$ & $34.18(7.03)$ & & $36.49(9.94)$ & \\
\hline Employed & $227(38.4)$ & $35.66(7.90)$ & & $38.78(\mid I .80)$ & \\
\hline Unemployed & $2(0.3)$ & $46.88(9.72)$ & & $56.87(\mid 3.26)$ & \\
\hline Missing & $25(4.2)$ & & & & \\
\hline Route of entry & & & 0.017 & & 0.013 \\
\hline Domestic transportation & $193(32.7)$ & $35.73(7.5 \mathrm{I})$ & & $39.11(11.86)$ & \\
\hline International transportation & $389(65.8)$ & $34.17(7.42)$ & & $36.71(10.39)$ & \\
\hline Missing & $9(1.5)$ & & & & \\
\hline Total & 591 & $34.73(7.48)$ & & $37.60(10.96)$ & \\
\hline
\end{tabular}

Abbreviations: T, days of quarantine; SAS, self-rating anxiety scale; SDS, self-rating depression scale; SD, standard deviation. 


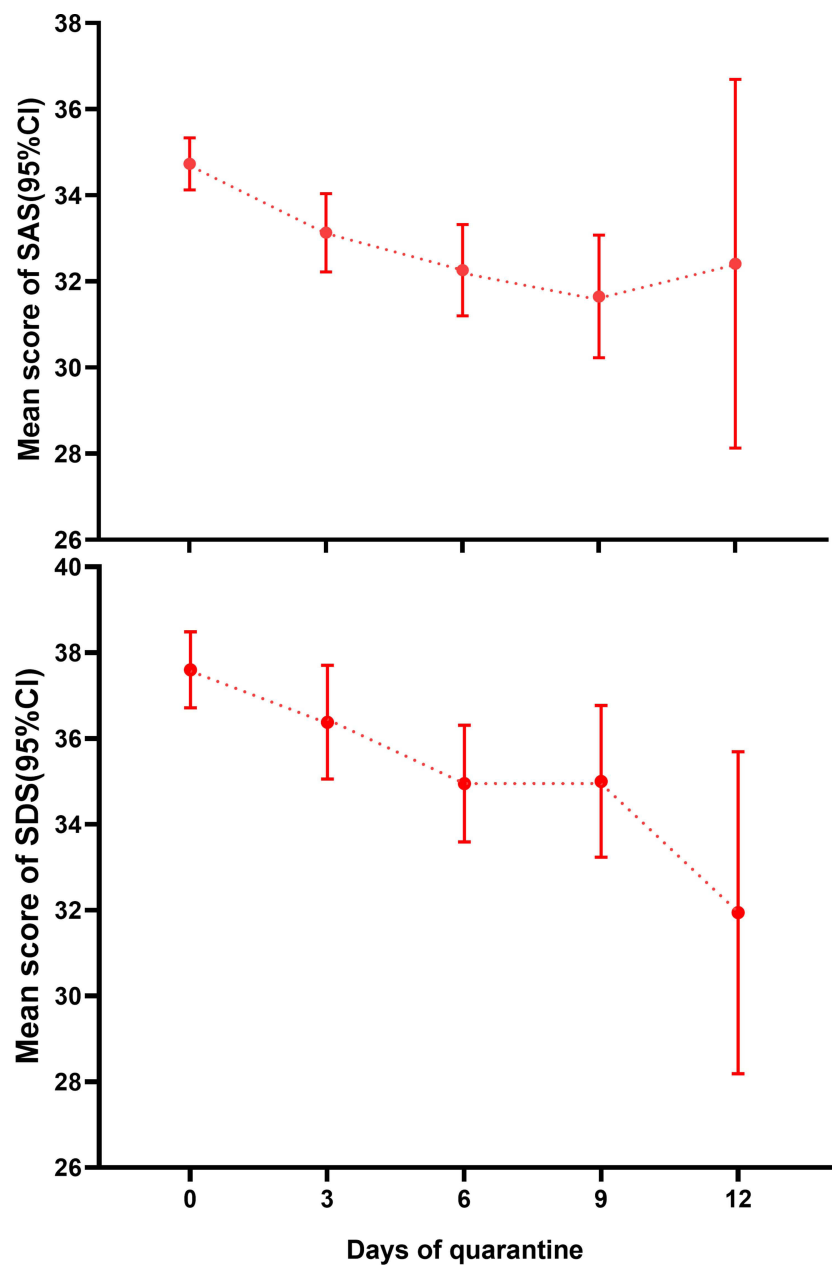

Figure 2 The trend of standard SAS and SDS scores of individuals during quarantine in dedicated hotels. The points marked in red represent the average scores of the questionnaires filled out at the recommended time points.

Abbreviations: SAS, self-rating anxiety scale; SDS, self-rating depression scale.

trend. The mixed-effects models were used to further explore the relationship between the days of quarantine (T) and the scores of SAS and SDS. Model 1 showed that T was negatively correlated with SAS score, and the square of T was positively correlated with SAS score, thus indicating that the SAS scale score first decreased and then increased with progressing of quarantine; the lowest SAS score appeared at tenth-time point $(\mathrm{T}=9)$. Meanwhile, participants aged 18 30 had higher SAS scores when compared with those $<18$ years old. Also, the unemployed had higher SAS scores compared to others.

Model 2 showed that the scores of SDS initially decreased and then increased with change of days of quarantine, while the lowest score of SDS appeared at the ninth time point $(\mathrm{T}=8)$. The influence of age and job on SDS scores was consistent with that of SAS scores.
Meanwhile, the later period of the quarantine date was associated with the higher SDS score, as shown in Table 2.

The interaction effect analysis revealed that under the current sample size, $p$ values for the test of heterogeneity of effect across subgroups were all $>0.05$, indicating that the changing trends did not significantly differ among subgroups, as shown in Table 3. To test the robustness of these results, the response time of some questionnaires that were not filled in according to the recommended date was adjusted, and the multi-level mixed-effects models were analyzed again. The results of these were consistent with those of the models without adjustment (Table A6 in Supplement).

There were 347 participants who answered the question about the causes of psychological distress and the possible mitigation way at the end of the quarantine. The results showed that the most common causes for psychological distress among participants were "fear of being infected by others" (35.4\%) and "maladjustment to the environment" (29.7\%). In order to alleviate their uneasiness, the participants mostly wanted to obtain information about "the nucleic acid test results" (53.6\%) and "specific time and arrangement for removing from the quarantine" (40.3\%) (Table A7 in Supplement).

\section{Discussion}

Unlike previous cross-sectional studies, this longitudinal study investigated the dynamic changes in symptoms of depression and anxiety during half a month of quarantine in individuals who were quarantined in dedicated hotels in China due to the COVID-19 outbreak. Our results revealed that the scores of psychological scales first decreased and then increased during quarantine. The scores were also affected by demographic factors such as age and job. With the aggravation of the epidemic, it is inevitable that a large number of people will be required to quarantine in dedicated hotels when traveling across the country. This study might provide a theoretical basis for the formulation of relevant policies that could alleviate the psychological distress of individuals during the quarantine period.

The incidence of depression and anxiety symptoms observed in the present study was lower compared to previous surveys that were conducted in the participants with quarantine during the infection pandemic such as SARS (7.6-31.2\%). ${ }^{15,17,25-27}$ We speculated that this might be because mortality rates of COVID-19 were relatively low compared with that of SARS (11\%). ${ }^{28}$ In fact, a similar low incidence of psychological distress was also 
Table 2 Longitudinal Change in SAS and SDS Scores by Increasing the Days of Quarantine

\begin{tabular}{|c|c|c|c|c|}
\hline \multirow[t]{2}{*}{ Variables } & \multicolumn{2}{|c|}{ Model I (Dependent Variable: SAS) } & \multicolumn{2}{|c|}{ Model 2 (Dependent Variable: SDS) } \\
\hline & Coefficient $(95 \% \mathrm{CI})$ & $P$ value & Coefficient $(95 \% \mathrm{Cl})$ & $P$ value \\
\hline \multicolumn{5}{|l|}{ Independent variables } \\
\hline Days of quarantine(T) (linear) & $-0.81(-1.00$ to -0.62$)$ & $<0.0001$ & $-0.75(-0.97$ to -0.53$)$ & $<0.0001$ \\
\hline Days of quarantine ${ }^{2}\left(\mathbf{T}^{2}\right)$ (quadratic) & $0.04(0.02$ to 0.06$)$ & $<0.0001$ & $0.04(0.02$ to 0.06$)$ & $<0.0001$ \\
\hline \multicolumn{5}{|l|}{ Confounding variables } \\
\hline Gender (ref=female) & $-0.43(-1.60$ to 0.74$)$ & 0.65 & $-1.84(-3.73$ to 0.06$)$ & 0.057 \\
\hline \multicolumn{5}{|l|}{ Age (ref=under I8) } \\
\hline $18 \sim 30$ & $3.17(0.52$ to 5.83$)$ & 0.019 & $3.98(0.14$ to 7.83$)$ & 0.042 \\
\hline $31 \sim 40$ & $1.93(-1.34$ to 5.20$)$ & 0.24 & $0.30(-4.45$ to 5.05$)$ & 0.90 \\
\hline $41 \sim 50$ & $0.87(-2.57$ to 4.32$)$ & 0.62 & $2.02(-2.97$ to 7.02$)$ & 0.43 \\
\hline $51 \sim$ & $0.33(-3.73$ to 4.40$)$ & 0.87 & $2.00(-3.89$ to 7.89$)$ & 0.50 \\
\hline \multicolumn{5}{|l|}{ Job(ref=student) } \\
\hline Employed & $1.53(-0.69$ to 3.76$)$ & 0.17 & $2.20(-1.03$ to 5.44$)$ & 0.18 \\
\hline Unemployed & $12.87(2.24$ to $23.5 \mathrm{I})$ & 0.018 & $18.00(2.87$ to 33.13$)$ & 0.020 \\
\hline \multicolumn{5}{|l|}{ Education(ref= Secondary school and below) } \\
\hline College degree & $1.66(-1.04$ to 4.37$)$ & 0.23 & $2.17(-1.76$ to 6.10$)$ & 0.28 \\
\hline University-Bachelor & $-0.4 \mathrm{I}(-2.15$ to $\mathrm{I} .35)$ & 0.65 & $-0.10(-2.65$ to 2.45$)$ & 0.94 \\
\hline University-Master and above & $1.56(-0.78$ to 3.91$)$ & 0.19 & $3.44(0.04$ to 6.84$)$ & 0.047 \\
\hline Route of entry (ref= International transportation) & $0.49(-1.20$ to 2.13$)$ & 0.58 & $1.84(-0.57$ to 4.26$)$ & 0.13 \\
\hline Sunlight in room (ref=Poor sunlight) & $-0.10(-1.27$ to 1.06$)$ & 0.86 & $0.72(-0.90$ to 2.34$)$ & 0.38 \\
\hline Period of responses & $0.18(-0.06$ to $0.4 I)$ & 0.13 & $0.39(0.06$ to 0.73$)$ & 0.020 \\
\hline
\end{tabular}

Abbreviations: SAS, self-rating anxiety scale; SDS, self-rating depression scale.

found in individuals quarantined due to the outbreak of H1N1 (mortality: 1\%). ${ }^{27}$

The results of multi-level mixed-effects models showed that the symptoms of anxiety and depression had a downward trend during the early period of quarantine, which might be due to the greatest psychological stress caused by the nervousness of people first coming to unfamiliar dedicated hotels. ${ }^{3}$ With the progression of quarantine, anxiety and depression were lessened in these participants. The possible reasons include: (1) if they were infected, they usually had symptoms a few days after the beginning of the quarantine; ${ }^{30}$ thus, with the progression of the quarantine, the risk of being infected decreases in these asymptomatic people; (2) they gradually adapt to the isolated living environment; (3) they have better access to medical services during quarantine because of the deployment of sufficient professional health personnel at the dedicated hotels, meaning that if they have any symptoms, they could be timely diagnosed and treated. Previous studies have also confirmed that sufficient material and medical services supply can effectively alleviate the psychological distress of quarantined people. ${ }^{3}$ These factors may also be the reason why some studies found no difference in psychological status between quarantined people and non-quarantined people. ${ }^{16,17}$ Our results suggested that when people first came to the dedicated hotels, they were the most in need of psychological counseling. Also, telemedicine and remote counseling may be a feasible way to provide psychological counseling, as it can reduce the risk of exposure to COVID-19. ${ }^{31}$

Moreover, we also found that the psychological symptoms of the participants deteriorated after 8 or 9 days of the quarantine. Previous cross-sectional studies reported similar findings, ie, the psychological symptoms of those in quarantine for $>10$ days were worse compared to those in quarantine for $<10$ days. ${ }^{25,26}$ Thus, the quarantine time should be controlled within 9 days if possible to reduce psychological distress caused by quarantine. As the incubation period of 2019-nCoV is less likely to exceed more than 9 days, converting the quarantine from dedicated facilities into home quarantine after 9 days of quarantine should be considered.

The unemployment and age 18 30 years old were associated with higher SAS and SDS scores, which was consistent with those of the previous studies. ${ }^{13,32,33}$ The unemployed and those who are 18-30-years old usually have poor economic conditions. Therefore, compared with 


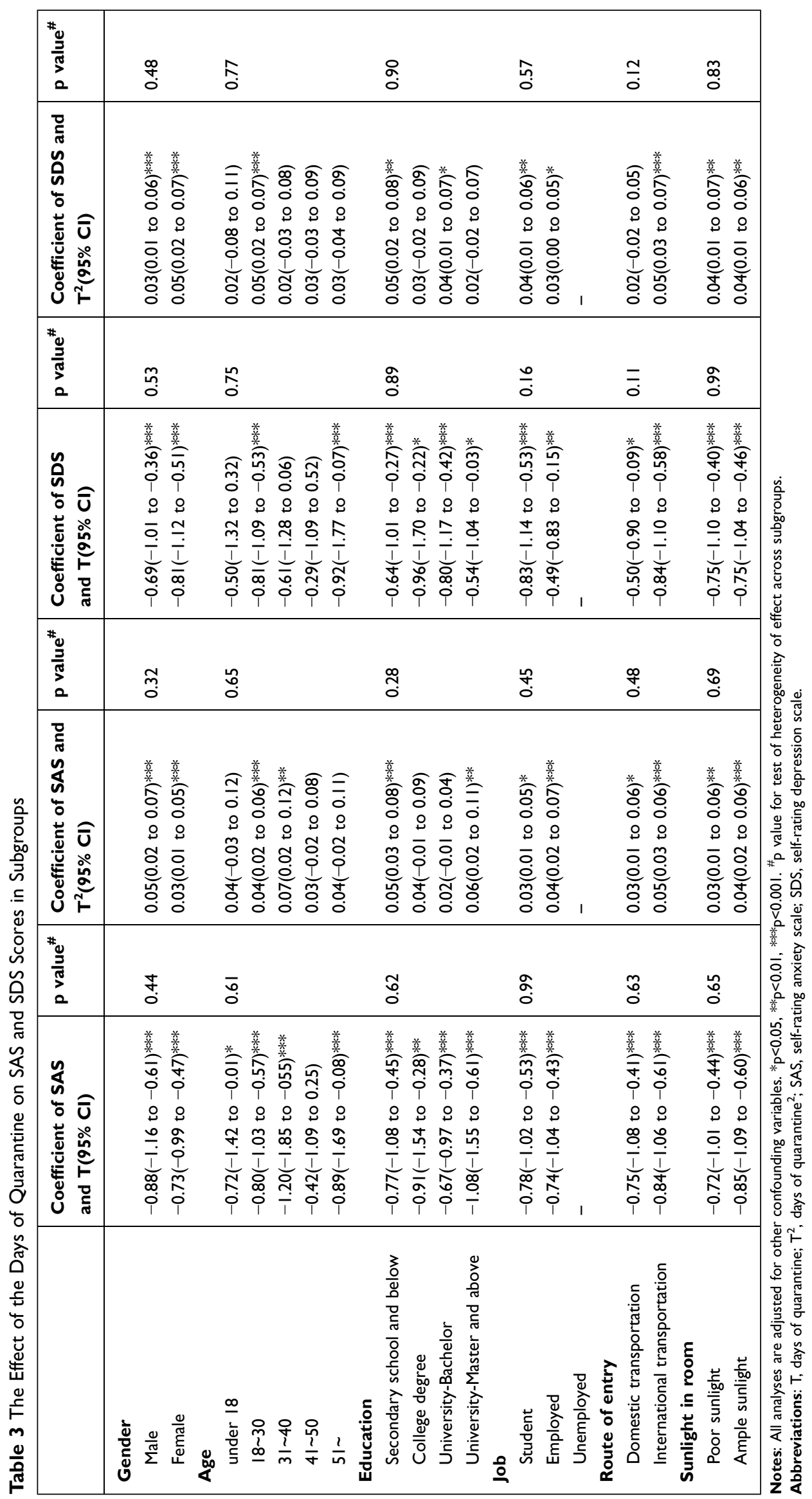


others, they have a weaker ability to resist risks and more worries about the future, which might lead to greater psychological distress. $^{3,13}$ Meanwhile, more depressive symptoms were observed in the later quarantine period, which might be because in March 2020, China stopped working, and people did not go to work regardless of being quarantined or not. ${ }^{12}$ However, the Chinese had gradually resumed their work since May 2020, but people who were quarantined in the later period could still not go to work and faced greater economic pressure, which might, in turn, lead to depression. Consequently, more attention should be paid to these high-risk subgroups.

The results of subgroup analysis showed no significant difference in the changing trend of mental health among subgroups during quarantine, including men and women, although women showed more psychological distress on the first day of participation in this study. Previous studies have also shown that gender greatly impacts psychological distress during quarantine, ${ }^{34,35}$ while further studies are needed to investigate whether there are any differences in the trends of their psychological status during quarantine.

According to the previous studies, stressors during quarantine include fears of infection, frustration, and boredom, and inadequate supplies and information. ${ }^{3}$ In this study, the most prominent stressors of psychological distress were "fear of being infected by others" and "maladjustment to the environment", which have also been identified in studies conducted during COVID-19 and SARS outbreak. ${ }^{25,36}$ The participants wanted to know about the "nucleic acid test results" and "the specific time and arrangement for lifting quarantine" to alleviate their anxiety. Previous studies have also shown that the spread of alarming information and fake news during the epidemic tends to aggravate the psychological distress of the population. ${ }^{37,38}$ Therefore, correct science popularization and education during isolation may help to reduce panic and ameliorate psychological distress. These findings also provide us with content that is relevant for psychological counseling.

The present study has some limitations. First, the time points when participants were filling out the questionnaire were not completely consistent. Some participants did not fill out the questionnaires according to the prescribed date (once every three days), showing an uncertain impact on data quality, although sensitivity analysis was used to reduce its impact on the results. Second, isolation measures during the outbreak limited the ability to follow up with these participants, and some participants filled out the questionnaire less frequently than they should. The underreporting of some people's mental health information may cause bias to the research results and reduce the reliability of the conclusion, although appropriate models were used to fully dig the information of existing data. Thus, further researches might be necessary to explore the trends in mental health during quarantine by higher quality followup data. Third, some factors that may affect the mental health of the population were not included in this study, such as emotional contact and pandemic awareness, ${ }^{37}$ but we believe that these factors have a limited impact on the dynamic changes in trends of mental health during quarantine.

\section{Conclusion}

This is the first study that revealed the dynamic changes in the mental health of the people quarantined in dedicated hotels during the quarantine period, suggesting that the mental health of the people entering from high-risk areas of COVID-19 coronavirus might be ameliorated during the early period of quarantine. Thus, if the quarantine in dedicated hotels is inevitable, the duration of quarantine should preferably be shorter. Also, the psychological status of the individuals should be closely monitored at the beginning of the quarantine and after more than 9 days. Meanwhile, more attention should be paid to unemployed individuals and those aged 18-30 years old. Future studies should include more variables that may affect mental health and conduct higher quality follow-ups of quarantined people.

\section{Data Sharing Statement}

The datasets analyzed during the current study are available from the corresponding author on reasonable request.

\section{Ethics Approval and Consent to Participate}

The local ethics committee of the Sixth Affiliated Hospital of Sun Yat-sen University has provided ethics approval. Written consent was obtained from all participants during the first section of the online survey before filling the questionnaire. If a participant was under 18 years old, then informed consent was obtained from respective guardians. The participants were fully informed about the purpose of the study and that it was conducted in accordance with the Declaration of Helsinki. 


\section{Acknowledgments}

All authors are indebted to the following professional bodies and their membership: the Guangzhou Center for Disease Control and Prevention, and the healthcare professionals in the dedicated quarantine facility. We wish to thank all participants and investigators for this survey. We are also grateful to all for giving valuable comments on this paper.

\section{Author Contributions}

WW, YD, and YJ designed the study. ZJ, CX, SL, ZC, YZ, and LK coordinated the study. LS and JL designed and ran the literature search. All authors contributed to data analysis, drafting or revising the article, gave final approval of the version to be published, agreed to the submitted journal, and agreed to be accountable for all aspects of the work.

\section{Funding}

The survey was supported by The National Natural Science Foundation of China [grant numbers 81974369 , 82041021]. The study sponsor had no involvement in study design, data collection, analysis, or interpretation of data.

\section{Disclosure}

All authors declare no competing interests.

\section{References}

1. Worldometer. COVID-19 CORONAVIRUS PANDEMIC; [updated 2021]. Available from: https://www.worldometers.info/coronavirus/. Accessed April 11, 2021.

2. Qiu J. Covert coronavirus infections could be seeding new outbreaks. Nature. 2020. doi:10.1038/d41586-020-00822-x

3. Brooks SK, Webster RK, Smith LE, et al. The psychological impact of quarantine and how to reduce it: rapid review of the evidence. Lancet. 2020;395(10227):912-920. doi:10.1016/S0140-6736(20)30460-8

4. Huang C, Wang Y, Li X, et al. Clinical features of patients infected with 2019 novel coronavirus in Wuhan, China. Lancet. 2020;395 (10223):497-506. doi:10.1016/S0140-6736(20)30183-5

5. Luo L, Liu D, Liao X, et al. Contact settings and risk for transmission in 3410 close contacts of patients with COVID-19 in Guangzhou, China: a prospective cohort study. Ann Intern Med. 2020;173 (11):879-887. doi:10.7326/M20-2671

6. News.gov.hk. Measures for travellers tightened; [updated 2020]. Available from: https://www.news.gov.hk/chi/2020/12/20201224/ $20201224 \_231616 \_358 . h$ tml type $=$ category\&name $=$ covid19\&tl $=\mathrm{t}$. Accessed January 2, 2021.

7. Huanqiu.com. Is the mining camp going to be an isolation point? Australia's strategy to prevent the spread of quarantine Hotel; [updated 2021]. Available from: https://baijiahao.baidu.com/s?id= $1688850482490238506 \& w f r=$ spider\&for $=$ pc. Accessed February 2, 2021.
8. CCTV news. Singapore tightens immigration control, requiring 14 days of mandatory quarantine for immigrants from Hong Kong. [updated 2020]. Available from: https://baijiahao.baidu.com/s?id= 1685839128303351380\&wfr=spider\&for $=$ pc. Accessed February 2, 2021.

9. Department of Health and Social Care. Guidance: how to quarantine when you arrive in England. [updated 2021]. Available from: https:// www.gov.uk/guidance/how-to-quarantine-when-you-arrive-inengland. Accessed March 10, 2021.

10. Immigration Department of Hong Kong Special Administtative Region of People's Republic of China. Statistics on passenger traffic. [updated 2020]. Available from: https://www.immd.gov.hk/ hkt/message_from_us/stat_menu.html. Accessed February 2, 2021.

11. Shi L, Lu ZA, Que JY, et al. Prevalence of and risk factors associated with mental health symptoms among the general population in China during the coronavirus disease 2019 pandemic. JAMA Netw Open. 2020;3(7):e2014053. doi:10.1001/jamanetworkopen.2020.14053

12. Tan W, Hao F, McIntyre RS, et al. Is returning to work during the COVID-19 pandemic stressful? A study on immediate mental health status and psychoneuroimmunity prevention measures of Chinese workforce. Brain Behav Immun. 2020;87:84-92. doi:10.1016/j. bbi.2020.04.055

13. Mollaioli D, Sansone A, Ciocca G, et al. Benefits of sexual activity on psychological, relational, and sexual Health during the COVID-19 breakout - ScienceDirect. J Sex Med. 2020;18(1):35-49. doi:10.1016/ j.jsxm.2020.10.008

14. Bai Y, Lin CC, Lin CY, Chen JY, Chue CM, Chou P. Survey of stress reactions among health care workers involved with the SARS outbreak. Psychiatr Serv. 2004;55(9):1055-1057. doi:10.1176/appi.ps.55.9.1055

15. Jeong H, Yim HW, Song YJ, et al. Mental health status of people isolated due to middle east respiratory syndrome. Epidemiol Health. 2016;38:e2016048. doi:10.4178/epih.e2016048

16. Dong R, Jiao X. Investigation on psychological status of isolation personnel during the outbreak of novel coronavirus. Rehabil Med. 2020;30(01):7-10. doi:10.3724/SP.J.1329.2020.01004

17. Zhu S, Wu Y, Zhu CY, et al. The immediate mental health impacts of the COVID-19 pandemic among people with or without quarantine managements. Brain Behav Immun. 2020;87:56-58. doi:10.1016/j. bbi.2020.04.045

18. Audit Bureau of Guangzhou. Give full play to audit function and contribute to epidemic prevention and control; [updated 2021]. Available from: http://www.zc.gov.cn/gzzcsj/gkmlpt/content/7/7336/ post_7336278.html\#3777. Accessed June 20, 2021.

19. Samakouri M, Bouhos G, Kadoglou M, Giantzelidou A, Tsolaki K, Livaditis M. Standardization of the Greek version of Zung's Selfrating Anxiety Scale (SAS). Psychiatriki. 2012;23(3):212-220.

20. Sakamoto S, Kijima N, Tomoda A, Kambara M. Factor structures of the Zung Self-Rating Depression Scale (SDS) for undergraduates. $J$ Clin Psychol. 1998;54(4):477-487. doi:10.1002/(SICI)1097-4679(199806)54:4<477::AID-JCLP9>3.0.CO;2-K

21. Zhou J, Yang Y, Qiu X, et al. Relationship between anxiety and burnout among Chinese physicians: a moderated mediation model. PLoS One. 2016;11(8):e0157013. doi:10.1371/journal.pone.0157013

22. Shen LL, Lao LM, Jiang SF, et al. A survey of anxiety and depression symptoms among primary-care physicians in China. Int J Psychiatry Med. 2013;44(3):257-270.

23. Wang Z. Chinese version of Zung's self-rating anxiety scale. J Shanghai Psychiatry. 1984;2:73-74.

24. Lee HC, Chiu H, Wing YK, et al. The Zung Self-Rating Depression Scale: screening for depression among the Hong Kong Chinese elderly. $J$ Geriatr Psychiatry Neurol. 1994;7(4):216-220. doi: $10.1177 / 089198879400700404$

25. Hawryluck L, Gold WL, Robinson S, Pogorski S, Galea S, Styra R. SARS control and psychological effects of quarantine, Toronto, Canada. Emerg Infect Dis. 2004;10(7):1206-1212. doi:10.3201/ eid1007.030703 
26. Reynolds DL, Garay JR, Deamond SL, Moran MK, Gold W, Styra R. Understanding, compliance and psychological impact of the SARS quarantine experience. Epidemiol Infect. 2008;136(7):997-1007. doi:10.1017/S0950268807009156

27. Wang $\mathrm{Y}, \mathrm{Xu} \mathrm{B}$, Zhao G, Cao R, He X, Fu S. Is quarantine related to immediate negative psychological consequences during the 2009 H1N1 epidemic? Gen Hosp Psychiatry. 2011;33(1):75-77. doi:10.1016/j.genhosppsych.2010.11.001

28. Chan KS, Zheng JP, Mok YW, et al. SARS: prognosis, outcome and sequelae. Respirology. 2003;8(Suppl 1):S36-40. doi:10.1046/j.14401843.2003.00522.x

29. Have T, Kunselman AR, Pulkstenis EP, et al. Mixed effects logistic regression models for longitudinal binary response data with informative drop-out. Biometrics. 1998;54(1):367-383. doi:10.2307/2534023

30. Li Q, Guan X, Wu P, et al. Early transmission dynamics in Wuhan, China, of novel coronavirus-infected pneumonia. $N$ Engl J Med. 2020;382(13):1199-1207. doi:10.1056/NEJMoa2001316

31. Tolone S, Gambardella C, Brusciano L, et al. Telephonic triage before surgical ward admission and telemedicine during COVID-19 outbreak in Italy. Effective and easy procedures to reduce in-hospital positivity. Int $J$ Surg. 2020;78:123-125. doi:10.1016/j. ijsu.2020.04.060

32. Wang Y, Yang YY, Li S. Investigation on the status of influencing factors for depression symptom of children and adolescents with home quarantine during the prevalence of novel coronavirus pneumonia. Chin J Child Health Care. 2020;28(03):277-280.
33. Li S, Wang Y, Yang L. Investigation on the influencing factors for anxiety related emotional disorders of children and adolescents with home quarantine during the prevalence of novel coronavirus pneumonia. Chin J Clin Med. 2020;28(04):407-410.

34. Platt C. Emotional and psychological distress related to COVID-19 isolation, quarantine, and physical distancing: evidence of gender-based differences. J Res Gend Stud. 2020;10(2):63-72. doi:10.22381/JRGS10220202

35. Buckner K. Gender-based emotional responses to perceived seriousness and fear of infection associated with COVID-19. J Res Gend Stud. 2020;10(2):73-83. doi:10.22381/JRGS10220203

36. Wu M Cai W, Wen T. Investigation of physical and mental health in isolated people during the outbreak of novel coronavirus pneumonia. Chin J Clin Med. 2020;27(01):36-40.

37. Lzroiu G, Horak J, Valaskova K. Scaring ourselves to death in the time of COVID-19: pandemic awareness, virus anxiety, and contagious fear. Linguistic Philos Investig. 2020;19:114-120. doi:10.22381/LPI1920208.

38. Bratu S. The fake news sociology of COVID-19 pandemic fear: dangerously inaccurate beliefs, emotional contagion, and conspiracy ideation. Linguistic Philos Investig. 2020;19:128-134. doi:10.22381/ LPI19202010
Risk Management and Healthcare Policy

\section{Publish your work in this journal}

Risk Management and Healthcare Policy is an international, peerreviewed, open access journal focusing on all aspects of public health, policy, and preventative measures to promote good health and improve morbidity and mortality in the population. The journal welcomes submitted papers covering original research, basic science, clinical \& epidemiological studies, reviews and evaluations,

\section{Dovepress}

guidelines, expert opinion and commentary, case reports and extended reports. The manuscript management system is completely online and includes a very quick and fair peer-review system, which is all easy to use. Visit http://www.dovepress.com/testimonials.php to read real quotes from published authors. 